\title{
Simulation of the ASFA system in an ERTMS simulator
}

\author{
I. Gómez-Rey, J. M. Mera \& A. Lorenzo \\ CITEF, Universidad Politécnica de Madrid, Spain
}

\begin{abstract}
Due to the economic, technical and functional advantages that the simulation tools of different railway signalling systems present and the possibility of driver training, an ASFA system simulator (Automatic Signalling and Brake Warning System) has been developed. This simulator includes on-board and on-track equipment. This system has been integrated in an ERTMS simulator allowing the generation of mixed scenarios as they happen on the new ERTMS lines that also use the ASFA safety system.

By integrating the ASFA system into another simulator both simulators are able to share several elements such as the virtual cab, applications for generating scenarios... but on the other hand, it has been necessary to develop new elements that simulate ASFA specifications. These elements have been implemented in $\mathrm{C}++$ and Component and Variables Register technologies have been used. The former allows us to divide the problems into less complicated modules that are assignable to real elements such as balises, signals, buttons... and the latter allows us to manage an exchange of variables among the different components that make up the system.

The ASFA components, which it has been necessary to generate since they are very different from those used in ERTMS, are the following. ASFA balises are devices that send information from the infrastructure to the train using the same messages defined in ASFA specifications; the antenna or sensor of the train has to receive the message from the balises and this message is transmitted to the processor unless some failure is simulated; the processor of the on-board equipment oversees the protection logic that the ASFA system develops; and the relay panel is the man-machine interface that shows the driver information relative to the state of the signals and the train, and which, in addition, allows the driver to realize certain actions and acknowledgements of the system.
\end{abstract}

Keywords: computer simulation, training, ERTMS, ASFA. 


\section{Introduction}

It is extremely useful to have a system that enables real situations to be simulated without having to face any of the actual risks that might arise, while at the same time reducing the investment costs required to build a real environment in which to perform the tests; an environment, however that does not totally eliminate the dangers or enable the specified requirements to be adapted to the necessary extent.

For several years, the railway industry has been using tools oriented towards testing train equipment in a controlled environment. In this environment, the test equipment is fed with the data that it is intended to receive from the other systems in the environment and its state and functionality is verified. It is also used for training the staff whose job will then be to drive the train or work with the real systems that are being simulated.

Bearing in mind these facts, different companies have developed railway systems simulators. CITEF (Centro de Investigación en Tecnologías Ferroviarias - Railway Technology Research Centre) in recent years has developed an ERTMS simulator for levels 0,1 and 2, which enables train circulation tests to be performed in a realistic way in already existing or planned infrastructures, without the need to physically run the trains on the lines, by simply launching the simulator [5]. One further step to complete the simulator is the implementation of the Spanish signalling system, ASFA.

\section{State-of-the-art}

SIFAV - RENFE [1]: In 2001 Indra, Tifsa and CITEF developed a training simulator for the Madrid - Seville high speed line that incorporates ASFA, LZB and ERTMS level 1 signalling for driver training.

Lander Simulation - RENFE [2, 3]: are developing a set of simulators for training drivers in situations of lack of safety and risk. They are fitted with ASFA, LZB and ERTMS that allow automatic operation and generate different situations for driver assessment.

Alten Ingeniería [4]: are developing an ASFA Digital train-track simulator.

\section{Objectives}

The objective of this project is to produce an ASFA simulator integrated inside an ERTMS simulator previously developed by CITEF [5]. This simulator will enable us to generate mixed infrastructures, that is, with ERTMS and ASFA components, just as is the case on actual lines, in such a way that depending on the system that is active on the train circulating on the line, it will either receive ASFA or ERTMS information.

The simulation embraces both infrastructure and on-train equipment. Therefore, different simulation scenarios can be simulated and the correct functioning of the train and the infrastructure can be verified as to mode changes, 
the level, the coherence of the messages sent by the balises, a despatch error can be simulated.

The simulator has been implemented in $\mathrm{C}++$ programming language by applying the component technology used for simulation in CITEF [6]. This enables each of the actual field elements to be represented as an individual component containing the input and output logic of the device represented.

\section{The ASFA signalling system}

The Automatic Signalling and Brake Warning System (ASFA) was designed by Dimetronic Signals to provide trains with greater operating safety. In a nutshell, its mission is for the train's driver's cab to show the phase of the signals that can be seen on the line, and condition drivers' actions to compliance with these signals' specifications and safety regulations Should signal requirements not be met, the automatic emergency brake is applied thus avoiding the risk of an accident.

The ASFA system includes the on-track and on-board equipment. The ontrack equipment's mission is to transmit the state of the signals of the direction of travel under simulation. The on-board equipment receives the information, records the driver's response and should the driver fail to respond, orders the emergency brake to be immediately applied.

The balise, which is the track element that transmits the information to the vehicle, is situated between the circulation tracks to the right of the centre axis of the track depending on the direction of travel. The balises are passive elements that transmit information to the train by means of inductive coupling with the train antenna when this passes over them. The message sent gives information on the state of the signal with which it is associated.

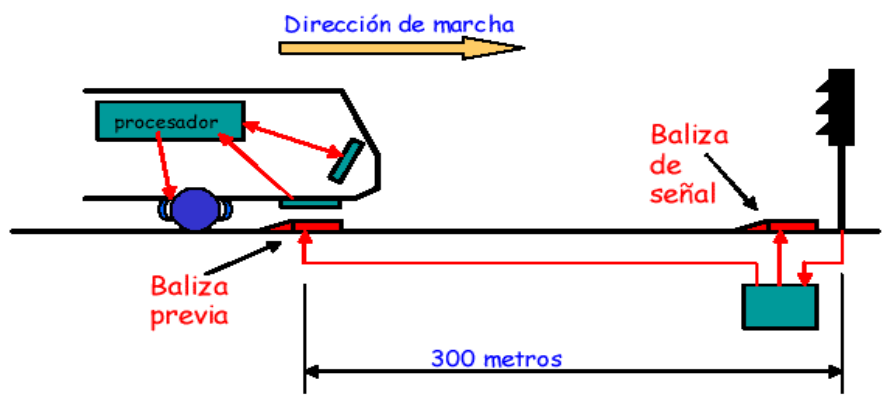

Figure 1.

The mission of the signals is to inform the driver visually of the number of free track circuits there are in the direction of travel. There are two balises associated with each signal: a preceding one (300 metres) and another at the signal base.

The messages sent to the train by the balise, depending on the state of the associated signal (it indicates the number of free signal overruns in front of the train) and balise type, are listed in the table below: 
Table 1.

\begin{tabular}{|l|l|l|l|}
\hline State of associated Signal & $\begin{array}{l}\text { No. Signal } \\
\text { Overruns Free }\end{array}$ & $\begin{array}{l}\text { Preceding } \\
\text { Balise }\end{array}$ & Signal Balise \\
\hline Flashing green or white & 3 or more & L3 & L3 \\
\hline Flashing green & 2 & L2 & L2 \\
\hline Yellow, yellow-green or bright yellow & 1 & L1 & L1 \\
\hline $\begin{array}{l}\text { Red, white, red-white or flashing red- } \\
\text { white }\end{array}$ & 0 & L7 & L8 \\
\hline
\end{tabular}

The information received from the train is shown to the driver via an ASFA relay panel fitted in the driver's cab. This panel is the man-machine interface and basically comprises buttons and an acoustic warning.

\section{Changes to the ERTMS simulator in order to simulate the ASFA system}

Below is a schematic diagram of the state of the ERTMS simulator prior to integrating the ASFA system. Shown next to this, are the components that show differences in behaviour in the ERTMS signalling system and the ASFA system.

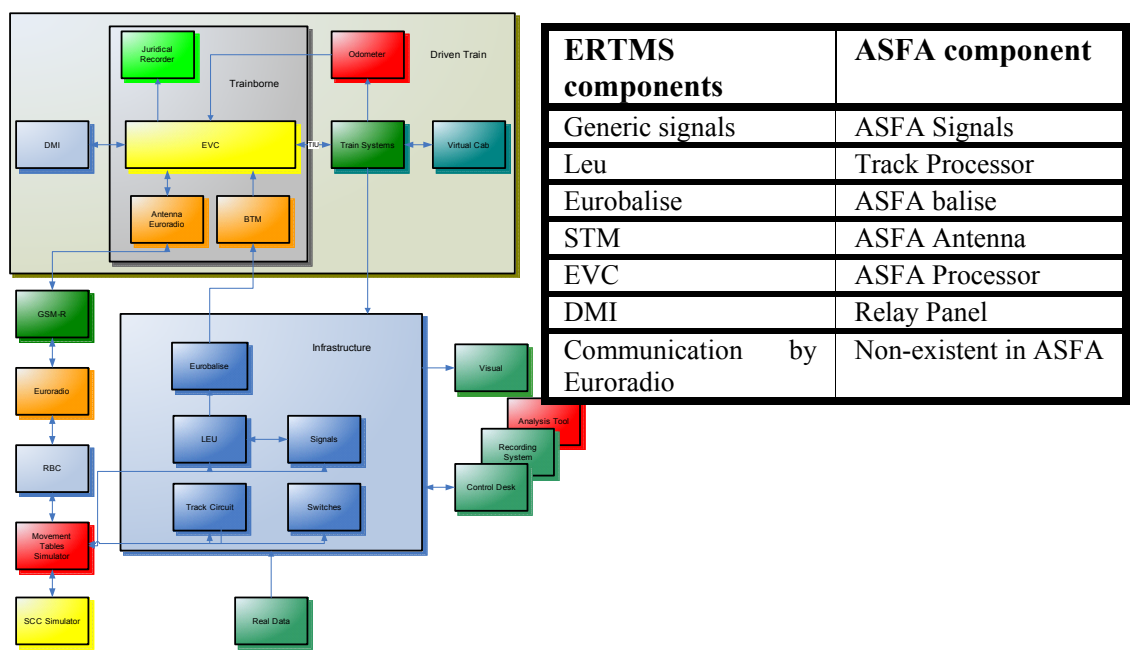

Figure 2.

In order to perform a simulation of the ASFA system the components shown in the table to the right have been changed or newly implemented, while the remaining components, those mainly related to the visual part of the simulator, train dynamics calculation, etc, have not undergone any variation.

Regarding the signals, the component used for ERTMS simulation is sufficiently generic since it allows all ERTMS signal lamps to be used: white, red, yellow, green, blue... so that the same component can be utilised by simply limiting the colours to be used to those used by ASFA signals. 
Likewise, the track processor used in ASFA is one of the functions used by the LEUs of ERTMS, so that, by using the signals' change of state logic according to the number of free track circuits these components can be reused in ASFA simulation.

On the other hand, the ASFA balises behave very differently from the eurobalises used in ERTMS, which means a new component has had to be implemented to integrate all the logic of both. Also, when mixed scenarios are generated, it will have to be indicated whether the balise is ERTMS or ASFA in order to insert one component or another.

However, regarding the on-board equipment, different ASFA components have been implemented: the antenna or sensor, the ASFA processor and the relay panel, which will be explained further on.

The first stage of the project was to develop the ASFA infrastructure equipment as simulation elements. To this end a study was undertaken of the devices that comprise a real ASFA infrastructure, how they interact with one another, their normal function and any possible malfunctions that might be of interest for simulation in the application being developed. This stage was completed by programming the new components needed to simulate the ASFA infrastructure and making the appropriate changes to existing infrastructure, thereby allowing us to load a mixed simulation scenario, that is, with both ERTMS and ASFA zones.

In order to integrate the ASFA system, changes have had to be made to the reader code of the files defining the infrastructure elements: signal overruns, track circuits, points ... so that when signals and balises are entered it will indicate if it is an ERTMS or ASFA element.

If the signals are ASFA, in order to differentiate them from ERTMS, all that need be done is to indicate which lamps the said signal can use and what their state will be depending on the number of signal overruns free in the direction of travel indicated.

To simulate ASFA balises, although their icon is the same, a different active component from that of the ERMTS balises has been implemented, whose logic will be explained below.

\subsection{BalizaASFA component}

This component is the active part that generically represents all ASFA balises of the installation: preceding or signal balises and it ensures compliance with their specifications.

The information needed to load these balises in the simulation, as well as to indicate that it is ASFA type, are the balise identifier, the balise group it belongs to, the section where it is located, and the kilometric point in this section (PK). By using the balise group identifier, the signal with which it is associated can be known together with the direction of travel in which the train receives its information and the balise type.

To sum up, the BalizaASFA component's mission is to observe the state of the associated signal, calculate the message to be sent to the train and send it 
when the train passes over the balise represented when the train's direction of travel is the same as that of the balise.

To simulate ASFA balise functionality, as well as communications between components, Variables Register [6] technology is used. This lets each component publish the variables of interest to other components in a list so that any component recorded can observe and establish the state of these variables. To be precise, the BalizaASFA component observes: the variable "state of the signal" and "direction of travel" published by the "signal" component whose identifier is that of the signal associated with that balise, and the "active section" variables and the "train Kilometric Point" published by the "infrastructure" component.

When BalizaASFA has been registered as the observer of these variables, the Variables Register takes charge of notifying the component when the state of the observed variables is changed, so that the Register can take the necessary measures: calculate the message to be sent, send this message... These functions are explained below.

The first function implemented is the one that calculates the message to be issued by the balise when the train is over it. This function has two input variables: the balise type (preceding or signal base) and the state of the associated signal. The first variable does not vary throughout the simulation as it depends on how the line has been configured to know if it is a preceding or signal base balise. The second variable is changed according to train circulation (as the different signal overruns are freed or occupied) as this directly influences signal state. Therefore, to calculate the new message to be sent, this function is called upon when the state of the associated signal is changed. Depending on these two variables and in compliance with the ASFA functionality indicated in the above section, the message is stored in an internal variable to be sent to the train when the train indicates that its PKs coincide.

Another outstanding function is the one whose mission is to check whether or not the train is over the specific balise that represents the component (there will be a BalizaASFA component for every balise on the simulated track). This function has various input variables: two referring to the balise and three to the trains in the simulation. Those related to the balise are established for the whole simulation: their position on the simulated line and the direction of travel of the associated signal which will be the direction the train will have to travel if the message is to be sent. The variables observed for each of the trains are: their position, direction of travel and if they are running under ERTMS or ASFA. This function will be called upon in every simulation cycle if the position of any train has been changed, so that by checking the train is over the balise, that the direction of travel is that corresponding to the associated signal and that the active system on the train is ASFA, then BalizaASFA will send the previously calculated message to the AntenaASFA of the corresponding train.

Another function simulates balise failure so that even if the train is passing over it, no information is sent to the train.

On the other hand, BalizaASFA also has to establish communications with the "AntenaASFA" components of each of the trains in the simulation so as to be able to send the message to the train over the balise. 


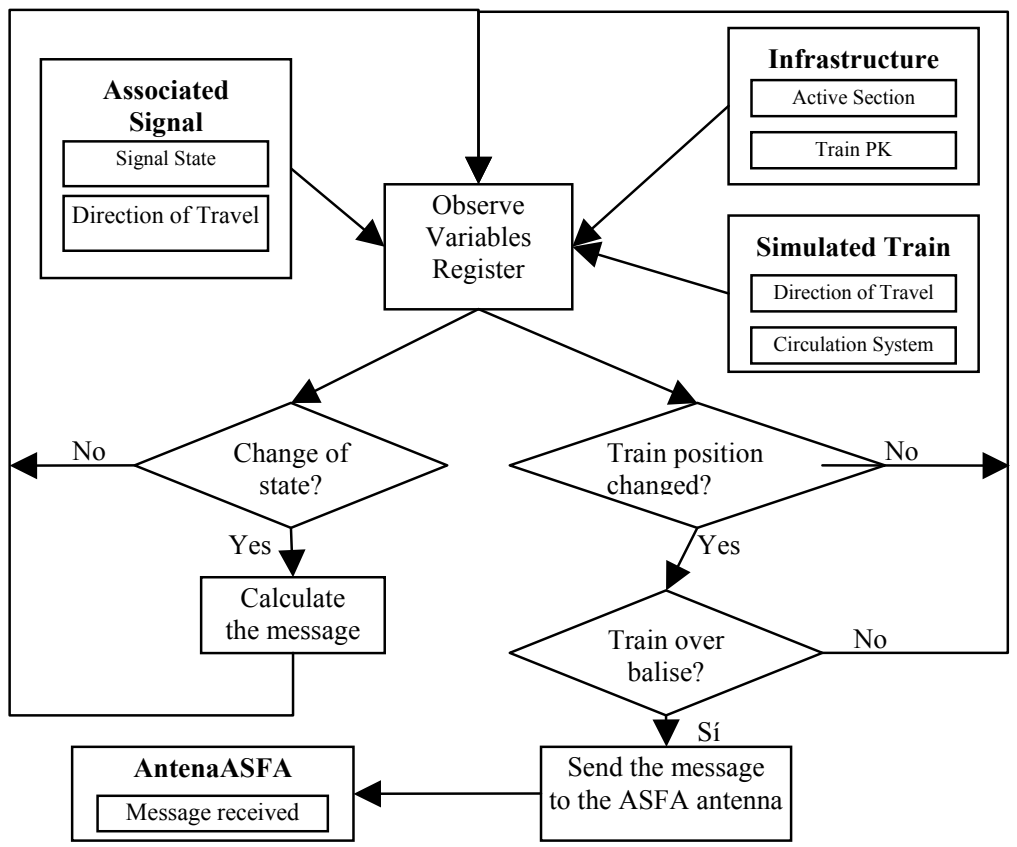

Figure 3.

Figure 3 is a schematic diagram showing the logic integrated into this component.

Finally, in order to be able to save and recover a previously loaded simulation scenario, these two functions are implemented using the same method as with the recording and recovery of ERMTS balises. Therefore, it is only necessary to include a new AsfaType variable to indicate if the balise is ASFA or ERTMS. To do this, this data must be entered into the existing Balise structure that contains all the fields needed to configure any infrastructure balise whatsoever.

\section{On-board equipment}

The second stage of the project was to produce all the components of the onboard equipment that enable the train to run on a track fitted out with ASFA and its integration into the virtual cab so that the train can run under ERTMS or ASFA (depending on how the system is started up). To be precise, the ASFA system will become activated when the simulator is started up on the STM level of ERTMS by selecting the Spanish ASFA signalling system.

The ASFA on-board equipment includes the following components: the antenna or sensor whose mission is to capture the message issued by the ASFA balises; the relay panel which is the man-machine interface and the processor whose mission is to control all these pieces of equipment, observe the 
information related to train dynamics and request the application of the emergency brake when necessary.

Below is an outline of how the different ASFA equipment components are related to one another and to other train equipment. These communications between different train components are carried out, as with the infrastructure, by using the Variables Register technology [6].

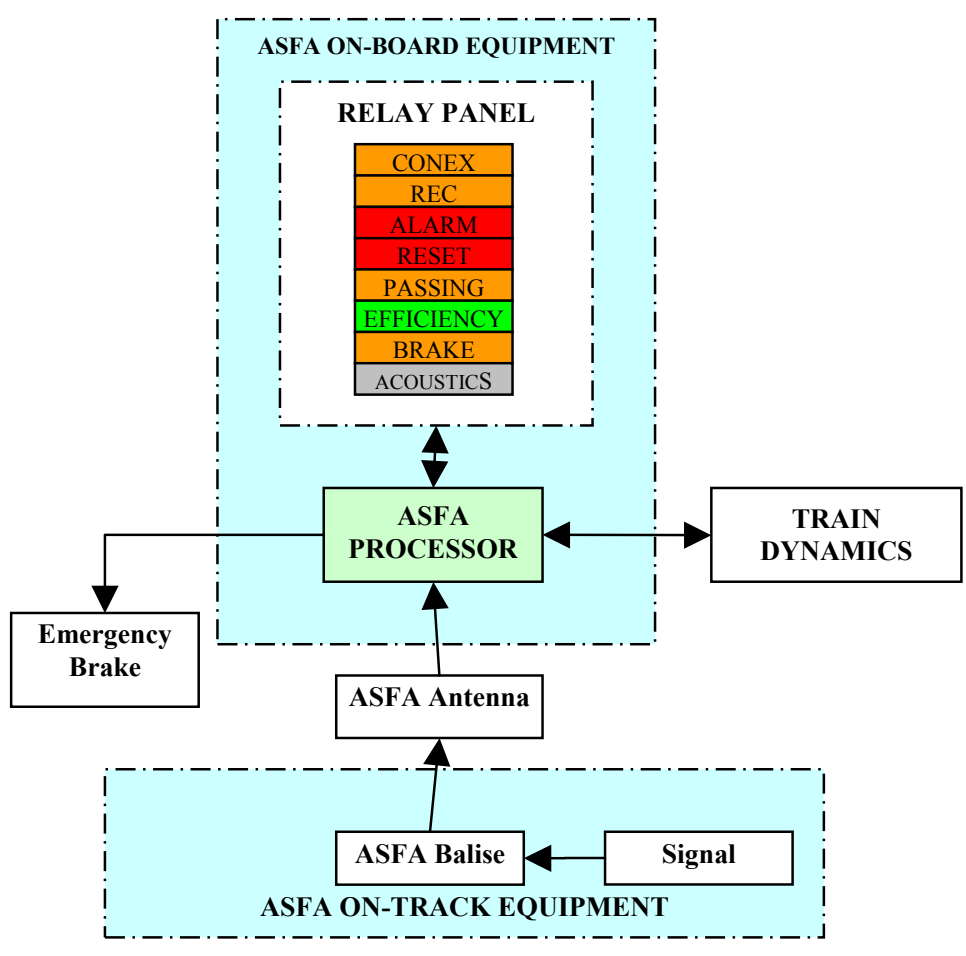

Figure 4.

\subsection{AntenaASFA component}

This component simulates the on-board equipment component whose mission is to receive the message sent by the ASFA balise and transmit this information to the ASFA processor so that it can take the appropriate measures. To achieve this, the "Message Received" variable is published in the Variables Register for each of the trains taking part in the simulation, so that the BalizaASFA components establish the value of this variable when the train passes over them. On the other hand, the ProcesadorASFA variable observes this variable so that it is notified when a new balise message is sent. 


\subsection{ProcesadorASFA component}

This component's mission is to store all the ASFA logic that determines the state of the buttons on the relay panel, the acoustic warning and the emergency brake in compliance with the specifications of the real equipment. To achieve this, it observes the information it needs from the different components making up the system by means of the Variables Register Container, processes the information and takes the appropriate decisions.

On the one hand, it receives track information from the antenna and on the other receives information from the on-board equipment: driver actions on the relay panel, train dynamics, if the train has been started under ASFA or under ERTMS... This component is also authorised to apply the emergency brake when safe operating conditions are not complied with: adequate speed, driver acknowledgement, correct state of on-board equipment...

Below is a schematic diagram with the basic algorithm integrated in this component.

A function has been implemented to simulate each of the possible balise messages that are called upon when these are received. As pointed out when

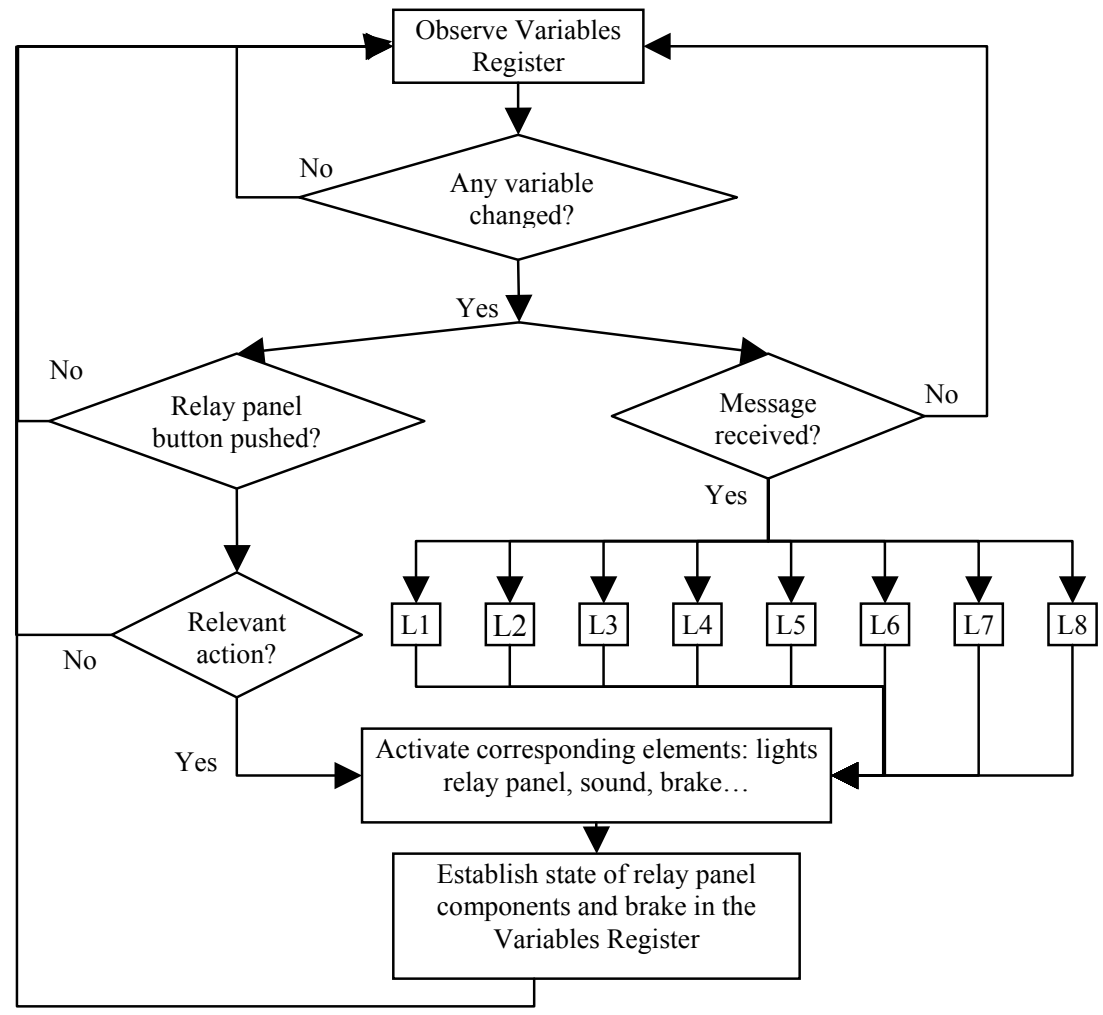

Figure 5. 
explaining the balises, messages L1, L2, L3, L7 and L8 are associated with a signal state and messages L4, L5 and L6 have been left in reserve for possible functions in the future. At present, should these reserved messages be received, the train emits a continuous acoustic warning for 3 seconds. Each of these functions reproduces the points indicated in ASFA systems specifications [7,8]: acoustic warning, corresponding relay panel indicators are lit, a maximum speed control is activated, driver acknowledgement is awaited should the message require such...

All these functions begin with an if condition, since from the start, the receipt of this message is only possible if the ASFA system is properly connected and the emergency brake has not been applied. If this condition is fulfilled, the value of the different on-board equipment elements that control the train is established.

In addition, a function has been included that that is run during each simulation cycle to control the time that passes between receipt of the message and the driver's pushing the button in acknowledgment, and to check that the speed is less than the speed imposed by the active control that applies the automatic emergency brake if the maximum permitted speed is exceeded as well as other secondary checks.

Other functions included are the alarm simulation and emergency braking, which are called upon when the conditions set out in the ASFA system specifications $[7,8]$ are complied with by activating the appropriate indicators.

\subsection{Relay panel}

The relay panel is the interface between the on-board equipment and the driver. It consists of a set of luminous and acoustic indicators that inform the driving cab personnel of the state of the track (next signal), the active speed controls, emergency brake application, state of the on-board equipment and other information of interest used by the ASFA processor, as well as some push buttons that allow the driver to acknowledge this information and perform other actions such as resetting the brakes, switching the desk on and off, authorising passing a red signal, etc.

Each relay panel element has been implemented with an independent component that will allow the functionality of each element to be dealt with separately: push-buttons, state of lighting, individual specifications of each button, etc. All these components have been included in a parent component integrated in the DMI (Driver Machine Interface), inside the virtual cab of the simulator, so that it remains hidden while the ASFA system is not active and is shown when the train is about to operate under ASFA.

The mission of each of the relay panel buttons, in general, is to inform the processor when the driver pushes them and observe their state (lit, switched off or flashing) according to what the processor tells them via the Variables Register. Moreover, these components have to observe the "DMI lit" and the "ASFA connected" variables since if one of the two is on zero, any buttons pushed on the panel will be ignored as the equipment will be switched off. Each of these components include a drawing function allowing an image to be associated (bmp) with the lit state and another with the switched off state, which all helps to make the simulator more realistic. 
Regarding the acoustic warning, the processor indicates if it is active or not and the time it needs to sound (it may be determined or undetermined). This component publishes a variable from the Variables Register that is observed by a "Sound Reproducer" component that plays WAV files at a volume indicated by this component. When this variable is zero the sound is not reproduced. With this philosophy the "acoustic warning" component observes the sound type that has to be reproduced and manages the variable of which we have spoken previously, giving it a high value during the time the ASFA sound has to be reproduced and zero when it has to be "without sound".

\section{Conclusions}

The ASFA railway system has been successfully and coherently simulated in an ERTMS simulator; that is, it can be used to simulate either of the two railway systems and even to generate scenarios containing infrastructures from both systems, as is the case with the new ERTMS lines. Moreover, it can be guaranteed that specifications $[7,8]$ are met as well as compliance with the functionalities of the real equipment.

This simulator allows an ASFA and ERTMS equipped line to be tested by inserting an ASFA level 0 train, for example, that "is following it", in such a way that it can be analysed if the ASFA train will encounter balises that limit its speed (due to the presence of the first train) which means it will be unable to travel the line at $200 \mathrm{~km} / \mathrm{h}$, and what running frequency would enable it to find all the signals on green: the lower the train frequency, the greater the running speed on the line, (without ASFA restrictions).

Other benefits of the ASFA simulator are that danger and failure situations can be generated along with other situations that actually arise, thereby making it possible to detect any black spots and ways to enhance safety.

Apart from technical simulations for enhancing line operation, the simulator can be used for training drivers that are to drive the locomotives fitted out with the ASFA-STD or ASFA-STD-200 system: the generation of different simulation scenarios where the train receives the different ASFA messages (normal driving), failure simulation of the on-board equipment or the balises as well as the functionalities offered by the ERTMS simulator: route setting...

Should such a request be made in the future, the simulator is prepared for real ASFA equipment that requires validation to be integrated, its interoperability with the simulated system being guaranteed.

Finally, it should be pointed out that thanks to simulation tools such as these, the costs of testing on real or planned lines are cut, as well as the costs of training drivers.

\section{Future developments}

The future developments that can be tackled thanks to this tool are the following:

- Automatic train simulation to include the Spanish ASFA model as only ASFA driven trains have been implemented. 
- To implement the transition of ERTMS to ASFA and vice-versa, treating ASFA as an STM level of ERTMS.

- The possibility of converting the ASFA simulator to the ASFA-digital system taking the components used for ASFA-STM simulation as a basis.

- In the future, possible integration of real components into the simulator to test and verify them.

\section{References}

[1] L.M. Gutiérrez, V. Giner, S. Tapia, C. Vera, J.M. Mera

[2] "Simulación de conducción y averías del tren Ave Serie 100"

[3] Actas del Congreso de Ingeniería del Transporte. CIT 2002. June 2002. Santander.

[4] LANDER SIMULATION - News from the 15-01-2007 http://www. landersimulation.com/index.php?id=13\&L=0\&tx_ttnews $\% 5 \mathrm{Btt}$ news $\% 5 \mathrm{D}$ $=57 \& \mathrm{cHash}=02 \mathrm{afb} 59 \mathrm{ed} 6$

[5] RENFE - Aulas Tecnologías de Conducción http://www.enpuntorenfe.es/ numero4/innovamos.htm

[6] ALTEN Ingeniería

[7] http://www.alteningenieria.com/\#05

[8] J.M. Mera, I. Gómez-Rey, A. Campos

[9] “ERTMS/ETCS test simulation bench". CompRail 2007

[10] A. Campos, J.M. Mera, I. Gómez-Rey, S. Tapia

[11] "Communication model for non-rigid interoperability between distributed applications". SISO 2005.

[12] Guía de Ayuda a la Conducción. Ave Madrid-Sevilla

[13] Author: ACTA-202.

[14] Documentación Técnica Sistema ASFA AVE.

[15] Author: Dimetronic, S.A. 